\title{
Collaborative Dynamics of Creative Teams: Modeling Creative Process in Advertising Design
}

\author{
Wisal Ahmad ${ }^{1}$, Mark Stufhaut ${ }^{2}$, Joe Labianca ${ }^{3}$
}

\begin{abstract}
Literature is very much clear about the role of teams in developing more creative outcome. However, question such as why one team produces greater creative outcome than others is very little addressed. This study addresses this question by looking into team dynamics in the context of advertising. More precisely, the study explores the creative process of advertising design by looking at how creative team members collaborate during the development of creative advertising in four advertising agencies using a qualitative comparative case study approach. Data was collected from teams comprising of creative directors, copywriters and art directors in four advertising agencies using semi-structured interviews followed by observation of the respective team members' interactions during the development of an experimental advertisement. Based on the creativity assessment of these advertisements by an independent jury of creatives, interview responses, and observation of the actual process of ad-design in four ad-agencies, the study findings reveal that the most creative advertisement follows a substantially much richer collaboration among the creative team members compared to least creative advertisements. In light of the actual interactions of the most creative advertising team, a preliminary model of creative advertising process is proposed that emphasizes on balanced configuration of domain competency of creative team members which plays a detrimental role in developing an interactional environment among creatives that leads to a creative advertising design. The study presents both theoretical and practical implications for better team management in organizations to produce more creative and innovative outcomes.
\end{abstract}

Keywords: Collaboration, Creative Process, Creative Advertising Design, Domain Competency, Interactional Environment.

\section{Introduction}

Advertising creativity plays a central place in the advertising world. The novel

1 Assistant Professor, Institute of Management Sciences, KUST. Email: wisalkust@hotmail.com

2 Professor, Gatton College of Business, University of Kentucky, USA.

3 Professor, Gatton College of Business, University of Kentucky, USA

\begin{tabular}{llll} 
ARTICLE HISTORY & & \\
\hline 6 Sep, 2017 Submission Received & 25 Sep, 2017 & First Review \\
\hline 20 Nov, 2017 Revised Version Received & 17 Oct, 2017 & Second Review \\
\hline
\end{tabular}

5 Dec, 2017 Accepted 
and creative idea provides a variety of benefits for both the clients and advertising agencies. Researchers argue that creative ideas can increase the sales of a company by five times (West, 1999). From the advertising agency's point of view, creative ideas can attract many new clients and the winning of creativity awards. The importance of advertising creativity can be well understood from the fact that each year, hundreds of creativity excellence awards are given to the most creative and outstanding advertisements (Till \& Baack, 2005). These awards are considered the barometer of performance in the advertising industry, which has significant implications for the clientele and recognition of an advertising agency and its employees.

Research on advertising creativity is, however, abstract as well as limited due to the abstract nature of creativity. Due to the ambiguous nature of creativity and hence the process that leads to such creative outcomes, creative process is not fully understood (Johar, Holbrook \& Stern, 2001; Till \& Baack, 2005.There are even considerable disagreements about the nature of the creative process, with some considering it an instantaneous moment of creation (Guilford, 1950; Wallas, 1926; Campbell, 1960), others considering the creative process as more structured and meticulous in which the mind produces creative outcomes by fulfilling structural boundaries like creative briefs, and time and budgetary constraints (Perkins, 1981; Weisberg, 1993; Dasgupta, 1994; ). Yet another view of the creative process states that it is in fact the combination of the earlier two approaches, and that creative process lies somewhere in between the freedom and constraints view of the creative process and believe that the process is unbalanced if there are too many restrictions or too much freedom (Hofstadter 1985). Nonetheless, creative processes are not fully understood leaving some key unanswered questions: "Are there processes which produce a more creative outcome?", and "Are these processes different for less creative outcomes?" (Woodman, Sawyer \& Griffin, 1993). Such questions are more relevant to advertising organizations where creativity is considered a central element for the organization's performance.

Much earlier, creative process has been conceptualized as the sum of sequential steps that an individual passes through while generating creative outcome (Wallas, 1926; Taylor, 1959; Young, 1976). These stages take a creative person from the stage of preparation through incubation, epiphany and lastly exposure (Wallas, 1926). Young (1976) considers the stages of ingestion and digestion instead of preparation stage suggested by Wallas (1926) and the rest of the process is similar to Wallas. According to Young (1976), a creative person has to catch a unique idea by following a narrow and straight linear approach across these steps. However, Bengtson (1982) has emphasized that either these stages are incomplete or not universally applicable (Vandern Bergh \& Stuhlfaut, 2006) as many brilliant ideas are not always the result of Youngs' model of creative stages (Rio Perez, 2006). As a matter of common sense 
and also supported by the researchers' community, these stages in the creative process are more of a generic form that all people may go through but produce different levels of creative outcomes (Kilgour, 2006). Moreover, previous research on advertising creativity, though much scarce, has looked into the creative process from individual perspective (Rio Perez, 2006; Vandern Bergh \& Stuhlfaut, 2006). This individualistic and unilateral approach to creativity implies that creative process is an isolated activity where creative individuals are not interacting with one another (Rio Perez, 2006). However, the current trend towards creativity research consistently points towards a more holistic and integrated approach of studying creativity (Hirschman, 1989, Johar et al. 2001; Rio Perez, 2006). Researchers argue that creativity in organizations is the result of a combined effort of a team where everyone contributes from their own respective domain. Barron (1999) while referring to this point used a biological metaphor that every individual is the creative product of two parents which Vandern Bergh and Stuhlfaut (2006) has termed collaboration. Hence, the current research attempts to adopt a broader teamwork perspective of studying the process of creative advertising design with the aim to address the unanswered questions of a more or less creative process that results in a more or less creative outcome (Johar et al., 2001).

Creative ideas are developed at advertising agencies by a creative team consisting of a copywriter, art director and creative director who collaborate on the task (Hirschman, 1989). Each one contributes to the development of the creative outcome from their own domain as well as helping one another mutually; hence, the nature of the creative process is more of collaboration. Researchers from several other disciplines including organizational behavior, information sciences and educational psychology stress that synergy is a prerequisite for creative outcome (McGrath, Arrow \& Berdahl, 2000; Young, 2000). However, little to no research has been conducted to study creativity in advertising from this teamwork/collaboration perspective (Johar et al., 2001). This study focuses on the creative process by looking at how a creative team collaborates during the development of creative advertising in a real advertising agency setting using a qualitative comparative case study approach. More precisely, the study draws from teamwork collaboration literature with a focus on Schrage dimensions of collaboration and investigates how creative team members collaborate during the process of creative advertising design.

\subsection{Research questions}

This research will primarily address the following main question:

1. How prevalent are the different dimensions of collaborative dynamics in the process of developing creative advertising? 
2. How the different dimensions of collaboration can be connected to develop a preliminary model of collaborative creative process in advertising agencies?

\subsection{Research objectives}

The primary objective of this research is:

1. To better understand the creative process in advertisements by looking at the collaborative dynamics of creative team members while designing a more or less creative advertisement.

To address the main objective of this study, the study seeks to address the following sub-objectives:

1.1 To understand the prevalence of different dimensions of collaboration in the development of a more or less creative advertisement.

1.2 To develop a preliminary model of collaborative creative process in advertising agencies.

\section{Literature Review}

Despite the widespread recognition of advertising creativity as a significant indicator of agency performance, the area of advertising creativity has not been adequately addressed and more surprisingly, research on the process of creative advertising development is then further limited and abstract. Even in the field of advertising, very little research has been carried out on the process of creative advertising design. Besides other factors, the complex nature of the creative process has been stated to be the most important factor for the lack of research. There is considerable disagreement about the distinctive nature of the creative process (Johar et al., 2001; Kilgour, 2006). Some researchers believe that the creative process is different than a routine and day to day thinking and it is mostly unstructured and thus cannot be measured. They believe creative process is analogous to a flash of light when the idea comes to mind (Guilford, 1950). They consider great freedom of thought and a lack of structural regulations as necessary for creative development. In contrast, reductionist researchers propose that the creative process is more meticulous and carefully thought out (Johar et al., 2001). Here creative idea generation occurs because of the mental constraints and thinking boundaries. They believe more in formulaic and structural views of the creative process. A third perspective is more integrative and suggests that creative process is a balance between freedom and constraints and that the process becomes unbalanced if there is complete freedom or complete constraints (Johar et al. 2001; Hofstdter, 1985). The difficulty with this perspective is, then, specifying where the 
balance point between freedom and constraint lies.

Because of such global differences about the nature of the creative process, researchers have turned to identifying and understanding its parts rather with the whole (Wallas, 1926; Taylor, 1959 cited in Johar et al., 2001). Much earlier, Wallas (1926) provided a four stage model of the creative process namely preparation, incubation, epiphany and exposure, which is widely acknowledged by advertising researchers. According to Young (1976), creative ideas emerge as a result of five steps covering ingestion, digestion, incubation, epiphany and exposure. Kover (1995 cited in Johar et al., 2001) studied the implicit theories of communication used by copywriters in the creation of advertising and formulated a post-hoc developmental sequence based on the copywriter's internal dialogue with an implied reader. Johar et al., 2001 studied the process of creating advertising by attempting to find a relationship between the creative process and creative advertising design. Hill and Johnsons (2004) suggest that creative process used in advertising agencies is verily standardized one and that most advertising is developed in a similar pattern of stages. Hence, this again does not answer the question of why one ad-agency produces more creative outcome than another agency. According to Stewart, Cheng, and Wan (2008), creative process consists of stages such as problem identification, deliberate thinking, illumination, evaluation and implementation. These stages resemble with that of Walls (1926; Young, 1976). Other researchers have also adopted the stages model of creative process (Griffin, 2008; Stuhlfaut \& Vanden Bergh, 2012). For instance, Griffin (2008) suggests four dimensions of the creative ideation process including orientation for the work, approach to the problem, mind-scribing and heuristics. The findings of one of the eminent researcher on creative process in advertising, Stuhlfaut and Vanden Bergh (2012) suggest that creative process in advertising is more of a metaphoric structure which includes perception, movement and object manipulation. Recent researchers are however of the view that while current research work in creative advertising process has provided some useful explanation of how creative ideas are developed in ad-agencies, the understanding of the nature of creative process within advertising agencies is limited (Turnbull \& Wheeler, 2015). According to Turnbull and Wheeler (2015), advertising researchers have still less understanding about the creative process that occurs in the development of a creative work particularly in advertising agencies. Moreover, very less research has appeared within the real-world environment of an advertising agency (Rio Perez, 2006). Rio Perez (2006) has given a conclusive view of the advertising creativity literature and states that during the entire period of 1972 to 2006 , creative process has been neglected and if studied, only the individualistic perspective of the creative process has been studied. The same concerns over the individualistic perspective of creativity study have also been stated by other recent researchers like Fourquest-Courbet et al. (2008), Courbet and Vanhuele (2008), Rio 
Perez (2006), Vander Bergh and Stuhlfaut (2006) and many others. This study begins with such an exploration of the process of creative advertising design by looking at the creative process from a teamwork interactions perspective. As stated by Chung (2009), little attention has been given to the creative synergy generated by teams during the creative process.

\subsection{Collaborative teamwork and creativity}

Creative outcomes are not the sole contribution of an individual; rather they are the outcome of a combined effort of the creative team. Chung (2009) states that the growing interest to explore creativity in inter-personal settings has led to a recent shift to study creativity from teams' perspective. As a result, the topic of teamwork and creativity has attracted research from several disciplines. Advertising researchers are of the view that there is a dire need to understand the creative process in advertising from multiple perspectives including team work collaborative perspective (Oliver \&Ashley, 2012; Vandern Bergh \&Stuhlfaut, 2006; Hirschman, 1989). While stressing the need for advertising creativity research, Hirschman (1989) states that creative ideas do not emerge in isolation, rather it's the outcome of social relationships that affect the process of creative advertising messages. The importance of collaboration was readily acknowledged in interviews with key creative personnel for the development of major and world level famous advertising campaigns for Levis Strauss (Champa, 1995b), Budweiser (Champa, 1996c) and MasterCard (Champa, 2000a).

Intellectual and artistic collaboration has been defined as "the interdependence of thinkers in the co-construction of knowledge" (John-Steiner, 2000, p.3). Reasons to collaborate include an increase in intellectual resource, improved results and emotional satisfaction (Kraut, Egido, \& Galegher, 1990). Smith and Arnston (1991) view creative collaboration as "a reciprocal, open dialogue which aims at a discovery of self and the transcendent nature of truth" (p.62) and a method for two people to achieve a state of experience beyond the material world. As an example, Smith and Arnston argue that the relationship between the artists Vincent Van Gogh and Paul Gaugin enabled each of them to achieve higher levels of creativity in their art than they would have been able to do alone. This in fact strengthens the proposition that high creative outcome (and hence a creative advertisement) is the result of team members' collaboration.

\subsection{Collaborative dimensions and team creative outcome}

As for the dimensions of collaboration against which team members' interactions could be evaluated, Smith and Arnston (1991) stated that the criteria for collaboration include genuineness, empathic understanding, positive regard for the other, presentness, and 
aspirit of mutual equality. Barron (2000) studied problem-solving groups and found that collaboration depends on the degree of mutuality, the amount of joint focus, and the degree to which the group members hold a collaborative orientation, rather than an individual one. Schrage (1995) presented the broadest set of criteria in regards to collaboration. Competence in domain, as evidenced in individuals is fundamental to the success of the collaborative team, shared and mutually understood goals, respect, tolerance and trust, space (physical dimension in collaborative relationships as the shared area whether it is a room, chalkboard or drawing table), representations of shared ideas, continuous but not continual communication, formal and informal work environments and clear areas of responsibility without strict boundaries.

The Schrage (1995) dimensions for creative collaboration is most extensive and covers almost all the dimensions of collaboration as stated by Smith and Artson (1991) and Barron (2000). For instance, "the positive regards for others, and spirit of mutual equality" dimensions of Smith and Artson (1991) are reflected by the "respect for tolerance and trust" dimension of Schrage, the presentness dimension is reflected by "representation of shared ideas, and continuous communication" dimensions of Schrage (1995). Similarly, the dimensions "the degree of mutuality, and the group orientation towards collaboration rather individual one" of Barron (2000) are reflected by the "shared and mutually understood goals" dimension of Schrage, the amount of joint focus dimension is reflected by "representation of shared ideas, shared and mutually understood goals, and continues communication" dimensions of Schrage (1995). In addition, Schrage (1995) also highlights the importance of other dimensions for creative and more functional collaboration including Competence in domain, clear areas of responsibilities but without strict boundaries, and the domain specific knowledge and competency of the team members. Hence, this study considers the Schrage model of collaboration as more comprehensive and encompassing for understanding the collaborative dynamics of creative team in advertising agencies.

\subsubsection{Competence in domain}

One of the foremost dimensions that Schrage states necessary for effective collaboration is domain-specific knowledge of creative team members. A collaboration of incompetents, no matter how diligent, cannot be successful (Schrage, 1995). A certain minimum threshold of competence is required for each member of the collaborative team. The knowledge of a domain refers to the knowledge base acquired to generate new ideas (Sosa \& Gero, 2007). Effective acquisition of a solid and diverse knowledge foundation has been stated to increase the chances of generating creative outcomes (Sosa \& Gero, 2007). Decades of research evidences that to a very large extent, success in the fields of music, sports, games and other complex domains reflects the knowledge and skills acquired through experience. This domain knowledge 
helps the creator to relate various concepts from his knowledge pool and transform it into a novel idea (Sosa \& Gero, 2007). Past research is undoubtedly clear that one of the largest sources of individual differences in peoples' performance on complex tasks is due to the factor of what and how much people know, that is, the declarative, procedural and strategic knowledge acquired through years of training and practice in their domain (Hambrick \&Meinz, 2011). Renzulli and Reis (1991) and Torrance (1962) emphasizes that past experience in creative involvement is a key indicator of creativity. Similarly, Ericson and Charness (1994) state that the effects of deliberate practice-engagement in activities specifically designed to improve performance in a domain are more far reaching than is commonly believed. Further, the domain knowledge including the basic knowledge and skills that individuals acquire (before and on the job) strongly correlate with the amount of working memory (Hambrick \& Meinz, 2011). Thus we believe that domain knowledge and competence (relevant formal education, trainings, and experience) would have positive effect on the creative performance of a collaborative group. More specifically, greater domain knowledge will reflect more creative outcome and vice versa.

Proposition: The domain competence of creative team members will have a greater positive influence on the creative performance of advertising creative team.

\subsubsection{Shared and understood goal}

The literature consistently highlights that one of the essential elements of successful collaboration is its focus toward a common goal and a clear purpose (Fisher, Hunter, \& Macrosson, 1997; Johnson \& Johnson, 2000; Harris \& Harris, 1996). This shared understanding of goal in fact creates a synergy, otherwise every member in the collaboration may not be able to contribute commonly and the resulting outcome may not truly reflect the aim of the collaboration, rather a combination of irrelevant pieces. The greater is this understanding of the project goal; the most relevant will be the contribution of each collaborator towards the whole. If a goal is ambiguous or ill-defined, the group will lack motivation and commitment. As Schrage (1995) states that the collaborators should be absolutely clear about what needs to be accomplished. They may tend to indulge in questions and answers about the task of how far along they (each member) understand the main goal of the task. Katzenbach and Smith (1993) have described five types of teams and how low performing teams differ than the high performing teams. The "working team, pseudo team and potential teams are low performers and their common reason is lacking common focus on the project goal. The "working group" consists of individuals who come together primarily to share information, best practices, and perspectives without a real set of group objectives. A "pseudo-team" is where a team may have a significant performance need but is not really trying to achieve it; and where there is no common purpose and as such the 
sum of its whole is less than the individual parts. A "potential team" is a team that is focused on an incremental performance need but there is a bias for reaching high performance. As a result, there is no accountability and mutual support for one another to achieve the group goal. A "real team" is a group of individuals who are equally committed to a common purpose for which they hold each other accountable (Regan, 1999). Yet another higher performing team is "high performance team" where the group members are not only committed to a common goal but also support one another for the combined success of the project.

Thus, we believe that in creative collaboration, the collaborators will be much clearer about what they are required to produce with greater amount of support for one another, and they will feel reciprocal accountability to one another since they will consider the failure of any one member as the failure of the entire collaboration. Based on this, the following proposition is developed.

Proposition: The sharing and understanding of advertising goal will have a greater positive influence on the creative performance of advertising creative team.

\subsubsection{Creation of shared space}

All collaborations rely on shared space. By shared space, Schrage (1995) means the collaboration tools used for visual display of ideas and thoughts including blackboards, project rooms, drawing tables or papers being passed between the collaborators at a coffee bar or lunch table. Whether physical or electronic, shared space is essential to successful collaboration as it provides a medium for communication or exchange of ideas. These shared spaces (physical or electronic shared space) stimulate thinking aloud and creating information together (Bredemeyer, 2002). The shared place should be equally accessible to all team members, dynamic and easily manipulable and capable of recording and preserving collaboration thought (Bredemeyer, 2002). Bredemeyer further clarifies that the best shared spaces stimulate the senses and the mind even when collaborators are separated by distance or time. Thus, we believe that the more shared space options (physical or electronic or both) are available to a collaborative team (creative team) for sharing of ideas, the greater will be the creativity of the outcome.

Proposition: A rich shared space in advertising agency will have a greater positive influence on the creative performance of advertising creative team.

\subsubsection{Representation of shared ideas}

Representation of shared ideas means in how many ways collaborators represent their ideas (Schrage, 1995). Collaborators usually fuse multiple perspectives of a task 
and hence they must use multiple representations for each of the perspectives. Schrage use the example of molecular biologists complementing their experimental data and discussions using computer models of organic structures. Similarly, theater directors rely on critical representations of text, actors' impressions, set designers, and possible audience response in order to fine-tune performances. Hence, greater representation of shared ideas is a significant element of effective and high performance collaboration.

In advertising agencies, creative team members typically express their rough creative concepts on a piece of paper or some other medium (shared spaces in this thesis) by using multiples perspectives. Other representations include photographs, typed copy or illustrations that all serve to express the ideas. They may even use multiple perspectives for a sub-task such as headline, story writing (the inherent drama in ad), and visualization. Since more representation of shared ideas about the task (i.e., the ad's drama, headline or tagline of the ad) helps in finding the most suitable idea that reflects the goal of the advertisement, the resulting outcome will be more representative of the collaborators. Hence, we believe that if the art director, copy writer and creative director use numerous perspectives to present their ideas, their collaboration will be more effective and unique.

Proposition: The greater and multiple representations of shared ideas between creative team members will have a greater positive influence on the creative performance of advertising creative team.

\subsubsection{Continuous but not continual communication}

Another dimension for successful collaboration is continuous communication (Schrage, 1995). Continuous communication keeps the collaboration alive and its group members active for the given task. Collaborators create a rhythm and a flow of communication appropriate to their relationship in which they continuously meet with one another and discuss the issue without a great time gap. This does not detach the collaborators from where they were discussing the last time they met compared to continual discussion in which there is a greater amount of interruptions and time gaps. Since there are no greater time delays in the task related communication, continuous communication is more task focused. Compared to communication with many interventions and time gaps, continuous communication takes the project to its end such that every member of the collaboration contributes effectively due to the avoidance of frequent interruptions. Based on this, it is proposed that creative teams with continuous communication will be more productive than ones where communication occurs frequently but with much more interruptions (continual communication). 
Proposition: The greater continuous (not continual) communication between creative team members will have a greater positive influence on the creative performance of advertising team..

\subsubsection{Operation in both formal and informal environments}

Creative collaboration and hence creative outcome occurs in multifaceted formal and informal environment. Schrage (1995) exemplifies that most of the creative outcomes in the field of art and science-from Niels Bohr to Vincent van Gogh, took place in informal environments like cafés and wilderness trips. Champa, as reported in a series of articles about creative inspiration, noted that creative advertising teams work in spaces that range from formal offices (1999b) to taverns (1991a) and airports (1995b). However, the formal setting has got its own importance where the need for a creative idea emerges (Schrage, 1995). In fact, working in both formal and informal environments, especially the latter, provides an opportunity for the collaborators to discuss freely, brainstorm and create many alternative ideas, which is one of the principles of creative idea generation. That is, during the creative idea generation process, creative people try to generate as many ideas as possible through association of various concepts that reflect the advertisement's central theme. The greater number of alternatives generated increases the likelihood that the resulting outcome will be more unique and creative. Thus, creative teams working in formal settings as well as in informal settings (e.g., break times in office such as lunch or tea breaks, arranging dinner at a restaurant etc) should lead to more creative outcomes compared to a creative team working on the advertising project only inside the advertising agency during official time.

Proposition: The greater involvement of creative team members in both formal as well as informal environment will have a greater positive influence on the creative performance of advertising creative team.

\subsubsection{Clear lines of responsibility but no restrictive boundaries}

The art director and copy writer in an advertising agency are responsible for the visualization of a creative idea and the verbal and written shape of the advertising idea respectively. Similarly, creative director supervises the entire creative project; however, it is not binding that creative ideas will always come from the art director, copywriter or creative director only. Though their responsibilities are divided functionally, anyone can make contributions in one another's areas during the creative process (Hirshman, 1989; Johar et al., 2001). From the perspective of Schrage (1995), what makes collaboration more creative and successful depends on how much one member consults, assists, and solicits ideas from the other member of the collaboration, irrespective of their functional positions. In other formal settings in some other 
organizations, such treatments or interactions may be considered more interference but in creative collaboration, there is little turf warfare because the collaborations are supposed to create one single collective solution to a given problem (Schrage, 1995). Thus, it implies that the greater is the reciprocal consultation and assistance process found in a collaborative group, the more the outcome will be creative. From an advertising perspective, we believe that the more a creative team is flexible in consulting and assisting one another in their functional areas (i.e., the copy writer assists the art director in the visual aspect of the idea, or the art director assists the copywriter in copywriting), the more creative advertising idea they will produce.

Proposition: The greater cross functional support among creative team members will have a greater positive influence on the creative performance of advertising creative team.

\subsubsection{Decisions are not made by consensus}

For an effective collaboration, the decisions may not only be made with simple consensus, rather there should be positive critique and argument-based reasoning for one another's ideas. This argumentative discussion is usually depersonalized since each member of the collaboration thinks from the perspective of the creativity of the end product, the success of which is the success of the entire collaborative group. The amount of argument-based reasoning depends on the number of genuine disagreements, how many perspectives and backgrounds each member uses, and the depth of discussion by the respective member in defending his point and idea (Schrage, 1995). It is important to note that collaborators, after argumentative discussion, agree on the direction they are taking, otherwise their collaboration would ultimately dissolve if the members consistently diverge (Schrage, 1995). Thus, it is not merely the consensus in its absolute term that makes an outcome less creative. What makes a consensus more effective is the way it is developed. If the consensus between the collaborators on an idea is arrived after logical discussion and sufficient background support aiming at the effectiveness of the final outcome, the consensus will be termed as more effective and the resulting agreement will contribute to more effective and higher quality outcome. Hence, the consensus thus arrived collectively based on objective ground will be a productive consensus resulting in a more creative and logical outcome than consensus reached for the sake of one another in any collaboration.

Proposition: The greater argument and objectives based decision making in a creative team will have a greater positive influence on the creative performance of advertising creative team.

\section{Methodology}

The primary objective of this study is to understand the process of creative advertising design by looking at the collaborative dynamics of creative teams to find 
out which type of collaborations produce more creative ads versus less creative ads. Based on this objective, this study takes a qualitative approach. To enhance the validity of the study findings, triangulation approach with a focus on semi-structured interviews followed by non-participant observation was used. Based upon literature review for team work collaboration and creative process, an interview protocol was developed. In order to provide a benchmark for understanding most creative versus least creative collaborative dynamics, a purposive sample of four advertising agencies with two agencies having won the advertising creativity awards and two agencies with no creativity awards was selected.

In order to collect the data, an oral permission was obtained from four advertising agencies. They were requested to participate in the study by designing a print advertisement which will be used only for academic purpose. For this purpose an advertising brief written by the researcher and checked by three creatives in two well known independent advertising agencies was given to each creative team in these agencies to develop a print advertisement. The brief asked for designing an advertisement for a newly launching mineral water brand that contains the extract of Nigella, (Chemically named as Black seeds of Sativa) which strengthens the immune system of body as well as useful in body healing. The brief included all pertinent information such as unique selling proposition (USP) of the brand, Target market and its specification, proposed personality of the brand, possible competitors etc. To collect data pertaining to teamwork interactions in the design of advertisement, a total of twelve semi-structured interviews from creative director, art director and copywriter were conducted in four advertising agencies respectively. This was followed by actual observation of advertising development process in general and the development of the experimental advertisement in particular, which took eight to twelve days in each respective agency. Once the experimental advertisements were developed, these were evaluated by each individual creative team member (art director, copywriter, and creative director) in an independent ad-agency using the novelty, meaningfulness, and relevancy measures of ad-creativity and its copy-visuals congruency (Ang, Leong \& Lee, 2007). Out of four such advertisements, one advertisement was assessed most creative whereas three ads were assessed more conventional and least creative. The findings of the study in terms of creative team members' interactions in each ad agency previously covered through interviews and observations during the development phase of their respective ads were then compared with the level of creativity assessed for their respective experimental ads. This led to the development of a preliminary model of collaborative creative process in advertising agencies. 


\section{Findings of the Study}

Based on the researcher interviews from creative team members followed by observations of the interactions of creative process in ad-design in four ad-agencies, the study finds varying level of team collaboration and interactivity in least creative and most creative ad-agencies. In the ad-agency whose advertisement was rated most creative, team members intensively interacted especially in the goal sharing and brainstorming stage. They used different perspectives to promote their respective ideas such as quoting local examples of herbal drinks; however, they also supported other members whenever a novel and catchy idea was presented. They were observed as having one unit without any impressions of supremacy and team domination. Further, in the rest of the ad-development process, team members remained in close contact through office telephone and email. The ad-copies designed by copywriter were sent to creative director and art director for the next day discussion on it. Similarly, ad-design was also discussed in team. Though, in these respective sessions, the respective creative member, being the expert of his domain, remained more active, however, in any such meetings, decisions were based on logical and objective discussion. Contrary, in the least creative agencies whose ads were rated less creative or much conventional, decisions were mainly personalized due to greater domain expertise of one of the team member. It would be right to say that even in the goal sharing and brain storming sessions in these agencies, communication remained unidirectional such that instructions were mainly coming from the senior creative member. The ultimate outcome and hence all such team decisions reflected one senior member thought. Even in the rest of the creative process such as copy of the ad or the ad design work, it was either an individual task of the respective team member followed by final approval by the senior creative member or the outcome reflected the dictation of the senior creative member. To sum up, the most creative agency remained much interactive in terms of goal sharing, cross functional support, multiple forms of representations and argument based decision making compared to least creative agencies where such dimensions were either not prevailing or in a much lesser intensity. Based on the findings of the study, a model of collaborative creative process is proposed.

\subsection{Modeling collaborative creative process}

Based on the interviews of creative team members including copy writers, art directors and creative directors in four advertising agencies followed by their interactions during the ad development process covering idea generation till the final ad, a preliminary model of collaborative creative process is proposed in figure 1 . Before describing the collaborative creative process model, it is important to assume that there is an underlying motivation purpose for creative team members that drive the collaborative creative process. The model depicts that during the different stages of 


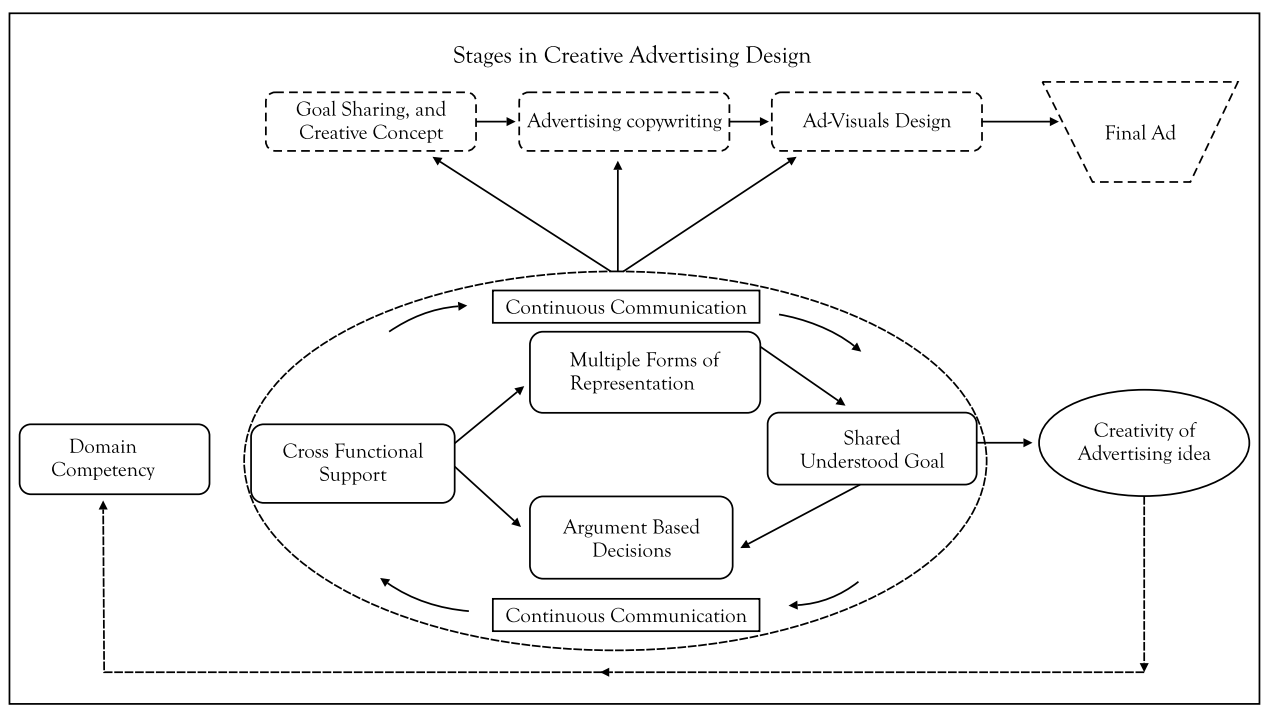

Figure 1: Collaborative Creative Process of Advertising

creative process such as brain storming sessions for idea generation, copy writing and art design, creative team members involve in cross functional dialogue using multiple forms of representation such as verbal discussion, demonstration such as the use of white boards and paper sketches etc for sharing of their views. This multiple representation by creatives not only foster creative process by enhancing team members' understanding and sharing of the team goal to be accomplished, but also serves as a guiding light for objectivity in decision making by collaborating members. The model also suggests that besides the indirect influence of creatives' multiple representations of ideas on objectivity in team decision making, it also directly results in teams' argument based decisions. This sort of collaborative environment covers both divergent and convergent thinking which is considered central to creative outcome.

Another very important aspect of the model is the greater potential of the cross functional collaboration to further the domain competencies and skills of the creative team members in the form of new knowledge learnt as a result of collaboration and this further accelerates the process of collaboration and creativity. This view is substantially supported in teamwork and collaboration literature (Schon, 1983; Finlay, 2009). Further, the observation data reveals that the stage of goal sharing and idea generation (creative concept development) covered comparatively much greater collaboration than in copywriting and ad-visuals design. Hence, the model indicates the intensity level of this greater interaction by three different arrows having a thicker arrow for creative concept development than other stages of the creative process. In addition, the two-sided arrows between copywriting and ad-visuals design indicate that this sequence may reverse in different times such that a creative concept may be 
captured first by advertising copy followed by ad-visuals and vice versa.

\section{Conclusion and Discussion}

The study findings reveal that creative team members in most creative advertising agency were possessing greater and balanced expertise in their respective domains which was found to play a determining role in establishing a conducive environment for cross-functional collaboration practices. It was found that during the preliminary sessions over the ad-brief understanding and idea generation, creative team members in the most creative agency were involved in a much involving and logical discussions over the advertising brief without any care or fear for their respective domains. They discussed different approaches, narrated their past experiences as well as possible social issues while suggesting a unique idea to present the advertising message. Creative team members used different ways to pass their message and make it more convincing and acceptable for other team members. For instance, this include verbal talk and demonstration. While discussing, they were trying to connect to and say "yes" or "no" to and disconnect with one another ideas. Contrary, in the least creative agencies, the study found that team members having imbalanced domain expertise with one member having high advertising knowledge dominated the others, and hence influenced the team interactions making it one sided such that the information was flowing mainly from the senior towards the junior creative team member. Decisions were mainly based on the inputs of senior creative member such that the other members were at the receiving end of the interaction. Overall, the interactive process in most creative agency was very much involving and objective with multiple forms of representations used by creatives compared to least creative agencies dominated by one or two creatives due to imbalanced configuration of team members domain knowledge, making the collaborative more subjective and less interactive. Based on the findings of the study, the study proposes a model of collaborative creative process in advertising design as shown in Figure1.

Literature on teamwork evidences that team members with their respective domain knowledge form a knowledge relationship system which affects the level and pattern of team members' interactivity (Schon, 1983; Quinn, 2000; Wasko \& Faraj, 2005). Hence, team members possessing high or low or imbalanced domain knowledge is more likely to have different effects on team members' interactivity and so on cross functional collaboration and team creativity. This study found that creative advertising teams in two of the least creative agencies were having imbalanced domain knowledge with one team member having high domain expertise and the other two members having less than one year domain expertise level. Contrary to this, team members with high and almost similar level of domain expertise were found highly interactive 
without any member dominating the team collaborative process. The findings of this study fully substantiate the views of Wasko and Faraj (2005) who found that people share and contribute their knowledge when they perceive that they have experience to share, and when it will enhance their professional reputation. Hence, it is concluded that when team members with high domain-related competencies collaborate and involve in a debate using multiple perspectives for a given task, it creates better understanding of team goal which serves as a benchmark for objective rather subjective based decision making and hence such team members' interactions generate more creative ideas.

With its remarkable findings on creative process, this study also has some limitations. Being the qualitative nature of the study, the superiority of the proposed model cannot be established without its empirical validation. Future research should therefore empirically test the model with a large sample size of creative teams in sufficient number of advertising agencies. Further, the organizational practices in Asian cultures significantly vary from those in other developed cultures such as Europe, America, and Germany etc. Hence, the study could be replicated in developed part of the world. Moreover, this study looked at the collaborative dynamics of creative teams while designing print advertisements. The nature of creative elements may vary in print and television advertisements such as the actual demonstration of creative message, advertising tone and jingles etc. which are not required for print advertisements. Hence, future studies should look into the team collaborative practices while designing a television advertisement.

\section{Limitations and Future Research Directions}

Though with the obtained evidence, the proposed model is plausible, however, as with all qualitative research studies, the superiority of the proposed model cannot be established without its empirical validation. Future research should therefore empirically test the model with a large sample size of creative teams in sufficient number of advertising agencies. To do so, it is suggested to preferably follow the methodological approach of Harvey and Kou (2013) due to its simplicity but more rigor in capturing team members' interactions. Second, the present study was conducted in four advertising agencies in Pakistan, a country of true reflection of Asian culture. The advertising industry of Pakistan and so its professional practices are still in infancy stage with comparatively low level of professionalism. Organizational practices in Asian cultures significantly vary from those in other developed cultures such as Europe, America, and Germany etc. Hence, it raises the question of transferability of such results to other such cultures. Future researchers should study the replicability of the collaborative creative process model proposed in this study in more developed 
cultures. Third, this study looked at the collaborative dynamics of creative teams while designing print advertisements. While the components of creativity may not vary, the nature of creative elements may substantially vary in print and television advertisements such as the actual demonstration of creative message, advertising tone and jingles etc in television advertisements which are not required for print advertisements. Hence, a creative team may require a richer collaboration to design a television advertisement. Thus, to understand the creative advertising process in a more integrated and holistic way, future researchers should study the creative teams' interactions while designing television advertisement. Fourth, as discussed above in detail, the proposition of greater domain expertise and cross functional collaboration was not supported in one of the least creative award-winning agency. Previous research on teamwork in organizations and creativity states that perception about the work environment affects teamwork (Goold, Craig \& Coldwell, 2008). Future studies should therefore also consider the effects of team members' perception about work environment on team members' motivation to engage in cross functional collaboration and its ultimate results on creativity.

\section{Acknowledgement}

I whole-heartedly thank Dr. Nancy Johnson, Dr. Joe Labianca, and my Postdoc research supervisor Dr. Mark Stuhlfaut for their consistent support and expert feedback during the entire period of this research. I am also much thankful to all the advertising professionals in Pakistan who enthusiastically gave me time in developing the experimental advertisements for this research. This research was supported by the KPK-University of Kentucky Business School Partnership Program (USA 2013).

\section{References}

Ahmad, W., \& Mahmood, Z. (2010). An empirical investigation of the association between creative advertising and advertising effectiveness in Pakistan. International Journal of Marketing Studies, 3(2), 32-52.

Ang, S. H., Lee, Y. H., \& Leong, S. M. (2007). The ad creativity cube: conceptualization and initial validation. Journal of the Academy of Marketing Science, 35(2), 220-232.

Bredemeyer, D. (2002). What it takes to be great in the role of enterprise architect. Bredemeyer Consulting. Enterprise Architecture Conference.

Buzzell, R. D. (1964). Predicting short-term changes in market shares as a function of advertising strategy. Journal of Marketing Research, 1(3), 27-31.

Chung, S. E. (2009). Supporting creativity in interdisciplinary teamwork: Examining relationships among individual traits, group characteristics, team process and creative performance in an applied setting. Unpublished Master dissertation, University of Florida. 
Dasgupta, S. (1994). Creativity in invention and design-computational and cognitive explorations of technological originality. Cambridge: Cambridge University Press

Finlay, L. (2009). Debating phenomenological research methods. Phenomenology E⿱ Practice, 3(1), 6-25.

Griffin, W. G. (2008). From performance to mastery: Developmental models of the creative process. Journal of Advertising 37(4), 95-108.

Hambrick, D. Z., \& Meinz, E. J. (2011). Limits on the predictive power of domain-relevant knowledge and experience. Current Directions in Psychological Science, 20, 275-279.

Hill, R., \& Johnson, L.W. (2004). Understanding creative service: A qualitative study of the advertising problem delineation, communication and response (APDCR) process. International Journal of Advertising 23(3), 285-307.

Hirschman, E. C. (1989). Role-based models of advertising creation and production. Journal of Advertising, 18(4), 42-53.

Johar, G.V., Holbrook, M.B., \& Stern, B.B (2001). The role of myth in creative advertising design: Theory, process and outcome. Journal of Advertising, 30(2), 1-25.

John-Steiner, V. (2000). Creative collaboration. New York: Oxford University Press

Kilgour, A. M. (2006). The creative process: The effects of domain specific knowledge and creative thinking techniques on creativity. Unpublished doctoral dissertation, University of Waikato.

Kurtz berg, T. R. (2000). Creative styles and teamwork: Effects of coordination and conflict on group outcomes. Unpublished doctoral dissertation. Northwestern University, Evanston, IL.

Nemeth, C., \& Nemeth-Brown, B. (2003). Better than individuals? The potential benefits of dissent and diversity for group creativity. In P. Paulus \& B. Nijstad (Eds.), Group creativity. (pp.63-84). Oxford: Oxford University Press.

Oliver, J. D., \& Ashley, C. (2012). Creative Leaders' Views on Managing Advertising Creativity. Journal of Marketing Theory and Practice, 20 (3), 335-348.

Osborn, A. F. (1963). Applied imagination (3 ${ }^{\text {rd }}$ ed). New York: Scribners.

Perkins, D. N. (1981). The minds best work. Boston: Harvard University Press.

Quinn, F. M. (2000). Reflection and reflective practice. In C. Davies, L. Finlay \& A. Bullman (Eds.), Changing practice in health and social care. London: Sage.

Rio Perez, J. D. (2006). Research in advertising creativity in the journal of advertising (1972-2006). Journal of Advertising, 35(3), 1-18.

Schön, D. A. (1983). The Reflective Practitioner: How Professionals Think in Action (Vol.5126). Basic books.

Schrage, M. (1995). No more teams! Mastering the dynamics of creative collaboration (2 ${ }^{\text {nd }}$ Ed). New York: 
Currency Doubleday.

Sosa, R., \& Gero, J. S. (2005). A computational study of creativity in design. AIEDAM, 19(4), 229-244.

Stewart, D. W., Cheng, Y., \& Wan, H. (2008). Envisioning the future of advertising creativity research: Alternative perspectives. Creative and effective advertising: Balancing spontaneity and discipline. Journal of Advertising, 37(4), 135-39.

Stuhlfaut, M. W., \& B. G. Vanden Bergh, B. G. (2012). Creativity is...: A metaphoric model of the creative thought process. Journal of Marketing Communications, 1(2), 1-14.

Taylor, I. A. (1959). The nature of the creative process. In P. Smith (Eds.) Creativity. (pp. 284-293). New York: Hastings House.

Till, D. B. \& Baack, W. D. (2005). Recall and persuasion: does creative advertising matter? Journal of Advertising, 34(3), 47-57.

Turnbull, S., \& Wheeler, C. (2017). The advertising creative process: A study of UK agencies. Journal of Marketing Communications, 23(2), 176-194.

Vanden Bergh, B. G., \& Stuhlfaut, M. (2006). Is advertising creativity primarily an individual or a social process? Mass Communication and Society, 9(4), 373-397.

Wallas, G. (1926). The art of thought. New York: Harcourt, Brace \& World.

Wasko, M. M., \& Faraj, S. (2005). Why should I share? examining social capital and knowledge contribution in electronic networks of practice. MIS Quarterly, 29(1), 35-57.

Weisberg, R. W. (1993) Creativity: Beyond the myth of genius. New York: W. H. Freeman.

West, D. C. (1999). $360^{\circ}$ of creative risk: An agency theory perspective. Journal of Advertising Research, 39(1), 39-50.

Woodman, R. W., Sawyer, J. E., \& Griffin, R. W. (1993). Toward a theory of organizational creativity. Academy of Management Review, 18, 293-321

Young, J. B. (1960). A technique for producing ideas. New York: McGraw Hill. 


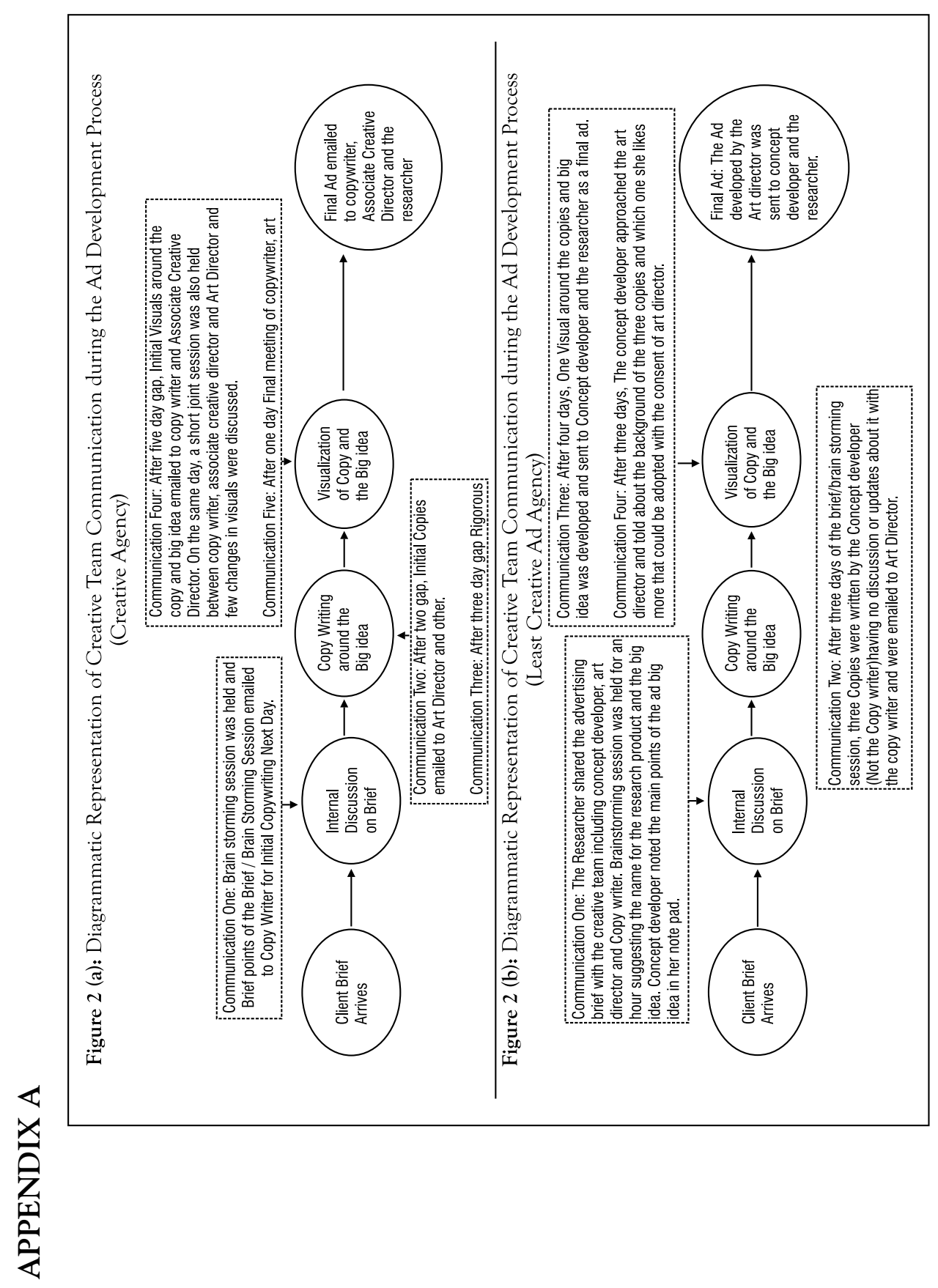




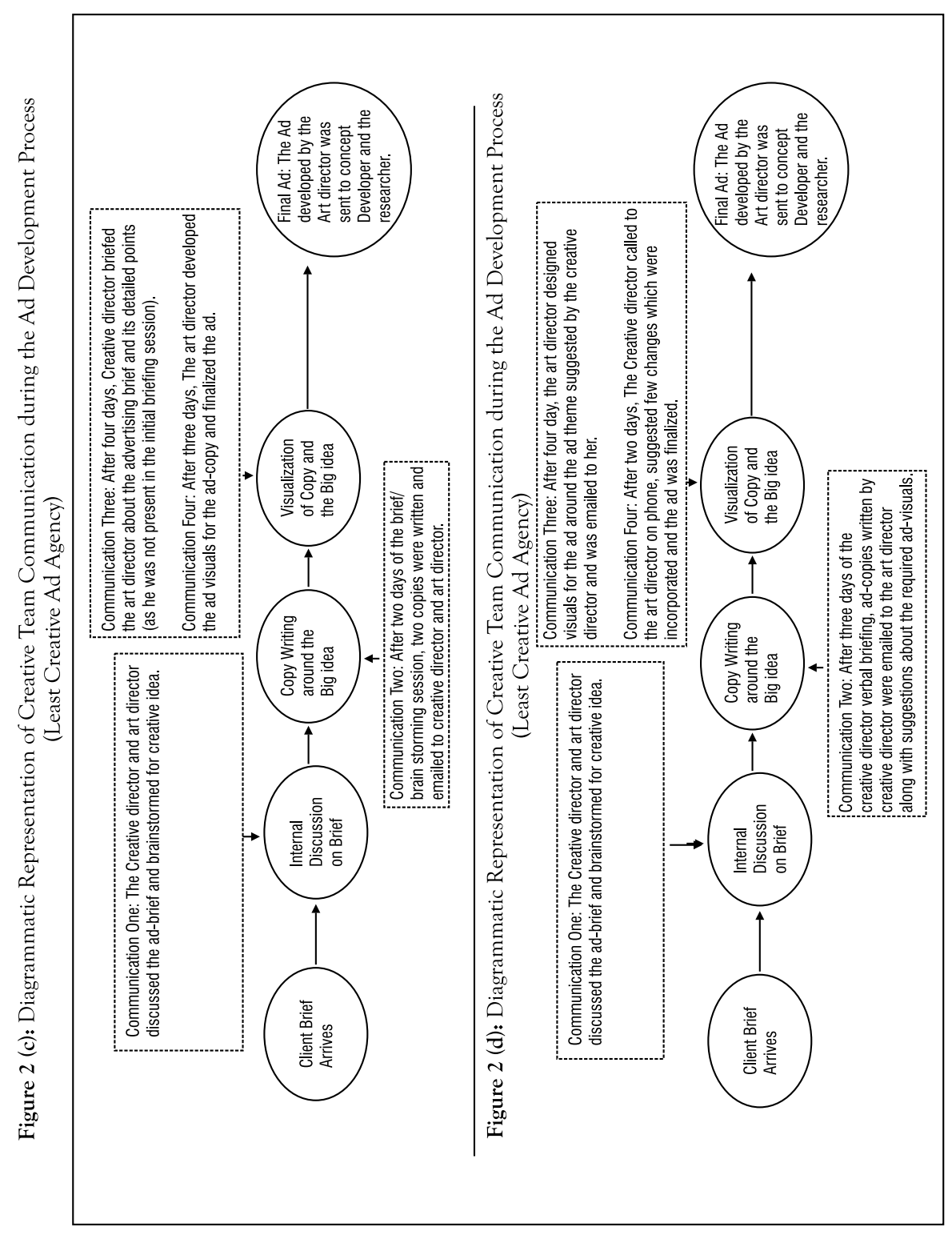




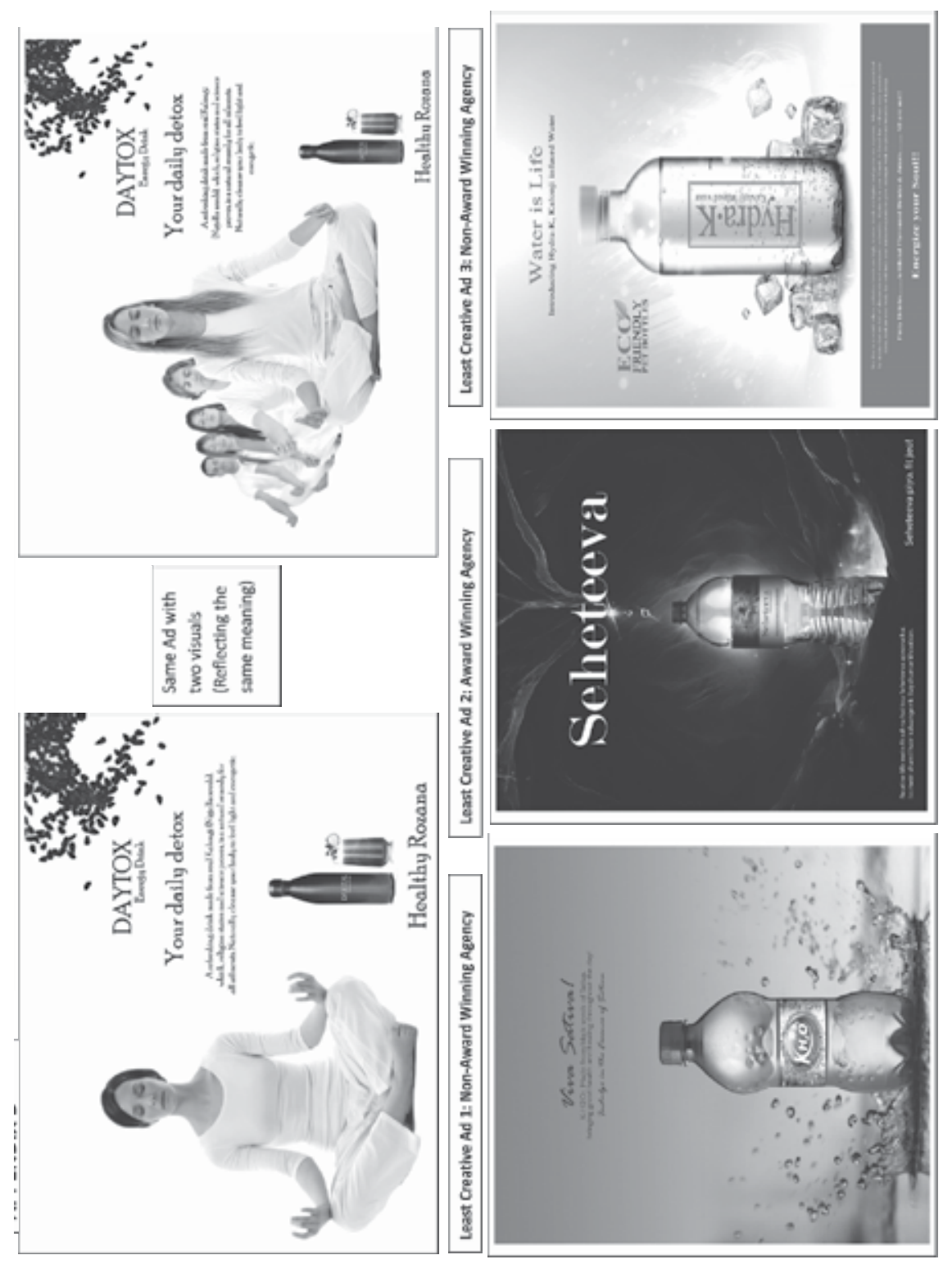


